\title{
Documentos
}

\section{Síndrome metabólico en la menopausia, conceptos clave}

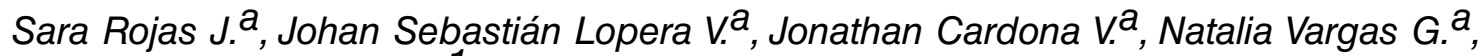 \\ María Patricia Hormaza A. 1 \\ ${ }^{1}$ Servicio de Ginecología y Endocrinología, Clínica Universitaria Bolivariana, Medellín, Colombia. \\ a Alumno/a, Facultad de Medicina, Escuela de Ciencias de la Salud, Universidad Pontificia Bolivariana, Medellín, \\ Colombia.
}

\section{RESUMEN}

La transición que experimenta la mujer durante la menopausia se asocia con el desarrollo de características propias del síndrome metabólico (SM), entre las que se encuentran: aumento de la grasa central abdominal, alteración del perfil lipídico y resistencia a la insulina. Por esta razón, la prevalencia de SM se incrementa con la menopausia hasta en un $60 \%$. La verdadera importancia de los factores que influyen en el riesgo cardiovascular en las mujeres posmenopáusicas está lejos de determinarse con exactitud y total certeza; estas pacientes deberían ser tratadas en forma temprana e integral con el fin de reducir su mortalidad.

\section{PALABRAS CLAVE: Síndrome metabólico, menopausia, enfermedades cardiovasculares}

\section{SUMMARY}

The transition experienced by women menopause is associated with the development of features of the metabolic syndrome (MS), among which include: increased core abdominal fat, abnormal lipid profile and insulin resistance. Therefore, the prevalence of MS is increased with menopause up to $60 \%$. The real importance of factors that influence cardiovascular risk in postmenopausal women is far from determined with accuracy and certainty; these patients should be treated early and comprehensive in order to reduce their mortality.

KEYWORDS: Metabolic syndrome, menopause, cardiovascular diseases

\section{INTRODUCCIÓN}

La menopausia, definida como el cese permanente de la menstruación, asociado a la declinación de la secreción de estrógenos por pérdida de la función folicular, marca así el final de la fase reproductiva en la vida femenina. Durante este periodo, el cuerpo de la mujer experimenta cambios físicos y emocionales como resultado a una serie de pro- cesos endocrinológicos, biológicos y clínicos (1-4). El déficit estrogénico propio de esta etapa se acompaña de un incremento en la incidencia de algunas condiciones médicas como la osteoporosis y las enfermedades cardiovasculares, lo que aumenta la mortalidad en este grupo etario (5-8).

La expectativa de vida de la mujer se hace cada día más prolongada, siendo para Colombia de 76 años aproximadamente; por esta razón 
cobra mayor importancia el estudio y el manejo de la menopausia, pues, teniendo en cuenta que su edad de aparición en nuestro medio es a los 50 años de edad aproximadamente, la mujer colombiana estará expuesta a este fenómeno hasta por un tercio de su vida $(9,10)$. Resulta entonces indispensable el abordaje de esta población por un grupo interdisciplinario de profesionales que valore su salud desde un enfoque integral, emprendiendo estrategias orientadas a la promoción y prevención de patologías prevalentes en la menopausia como el síndrome metabólico, con el finde disminuir el riesgo cardiovascular, y con esto, lograr impactar en la morbimortalidad de estas mujeres (5-8).

\section{FISIOLOGÍA DE LA MENOPAUSIA}

La menopausia es una etapa que está marcada por el inicio de la declinación de la función ovárica, motivada por el paulatino agotamiento o atresia folicular, lo cual provoca a la larga la desaparición de la ovulación y de folículos ováricos que puedan responder a las gonadotropinas; esto se asocia entonces a la falta de producción de hormonas sexuales femeninas como los estrógenos y la progesterona $(5-8,11)$.

Típicamente, se ha identificado a la estrona como el estrógeno más importante en la posmenopausia. Este es el resultante de la conversión a nivel de los tejidos periféricos de la androstenediona (12-16). Por otro lado, los niveles de hormonas como el estradiol son muy bajos, con un aumento marcado en las concentraciones de FSH y en menor medida de la $\mathrm{LH}$, de tal forma que el cociente $\mathrm{FSH} / \mathrm{LH}$ se invierte y es mayor de 1. Es por esta razón que los valores de FSH por encima de 40 $\mathrm{UI} / \mathrm{L}$ son compatibles con la interrupción completa de la función ovárica $(3,4,7,8)$.

\section{GENERALIDADES DEL SÍNDROME METABÓLICO}

El síndrome metabólico (SM) es un término que describe un conjunto de factores de riesgo independientes que aumentan la probabilidad de enfermedad cardiovascular; hace referencia a una condición causada por la obesidad abdominal que incluye hipertensión, diabetes y dislipidemia $(17,18)$. A través del tiempo ha recibido también otros nombres como síndrome $\mathrm{X}$, síndrome de resistencia a la insulina y el cuarteto de la muerte (19). EI SM se está convirtiendo en una epidemia mundial, el aumento global de su prevalencia, que está muy extendido en los países tanto industrializados como en desarrollo, es el resultado de una mayor proporción de obesidad y sedentarismo en la población (17-19).
El aumento del riesgo cardiovascular en el SM es el resultado de una compleja interacción entre los factores de riesgo individuales que aún no se entiende completamente (20). Por ejemplo, aunque la obesidad central es una característica definitoria de SM, un estudio en hombres de mediana edad con este síndrome encontró que el riesgo cardiovascular se incrementó independientemente del índice de masa corporal (21); además, una asociación de mayor riesgo de enfermedad cardíaca isquémica, así como de ictus isquémico en pacientes con SM fue observada en personas menos obesas (22). Otro estudio encontró que los pacientes con este síndrome que también presentan disfunción endotelial se encuentran en un mayor riesgo de enfermedad cardiovascular (20). Esta evidencia ha permitido concluir entonces que el SM aumenta el riesgo de enfermedad cardiovascular en una medida mayor que la probabilidad conferida por cualquiera de sus componentes individuales (20-22).

Han sido identificados cuatro elementos que comprenden el SM: obesidad central, dislipemia (aumento de triglicéridos y reducción de lipoproteínas de alta densidad y colesterol), hipertensión arterial e intolerancia a la glucosa; sin embargo, las definiciones utilizadas varían un poco entre los grupos étnicos y los criterios diagnósticos han sido establecidos por organizaciones diferentes con ligeras variaciones en los mismos (Tabla I). Otros factores tales como estados proinflamatorios y protrombóticos también se han asociado al SM (23-25).

EI SM puede estar presente en formas diferentes, de acuerdo a la combinación de los diferentes componentes del síndrome, y está bien establecido que aumenta el riesgo para el desarrollo de enfermedades cardiovasculares, diabetes tipo 2 y cáncer $(19,28)$. Todavía no se sabe cómo el SM es activado o cómo los diferentes componentes tienen una relación causal, pero la resistencia a la insulina, se sospecha fuertemente, posee un vínculo fisiopatológico común (29), ya que es evidente que existe una correlación positiva entre el peso corporal y la resistencia a esta, además del riesgo de desarrollar las anormalidades metabólicas asociadas $(30,31)$. Sin embargo, datos recientes sugieren que el SM y la obesidad no siempre se producen en concordancia (32); por ejemplo, algunos estudios sugieren que la obesidad franca no se traduce necesariamente en resistencia a la insulina y aumento del riesgo de comorbilidades metabólicas. En un estudio transversal de 5440 participantes de la Encuesta Nacional de Examen de Salud y Nutrición 1999-2004, el 31,7\% de los adultos obesos (IMC $\geq$ 30) eran metabólicamente sanos (33). 


\section{CRITERIOS PARA DIAGNÓSTICO DE SÍNDROME METABÓLICO SEGÚN NCEPIATPIII E IDF}

\begin{tabular}{|c|c|c|}
\hline Criterio & $\begin{array}{l}\text { NCEP/ATPIII } \\
\text { SM si: tres o más de los siguientes } \\
\text { criterios }\end{array}$ & $\begin{array}{l}\text { IDF } \\
\text { SM si: obesidad abdominal más dos de los } \\
\text { siguientes criterios }\end{array}$ \\
\hline Obesidad abdominal & $\begin{array}{l}\text { Circunferencia de cintura mayor de } \\
88 \mathrm{~cm} \text { en mujeres y } 102 \text { en hombres }\end{array}$ & $\begin{array}{l}\text { Circunferencia cintura } \geq \text { de } 80 \mathrm{~cm} \text { en mujeres y } \\
90 \mathrm{~cm} \text { en hombres }\end{array}$ \\
\hline Triglicéridos & Mayor o igual a $150 \mathrm{mg} / \mathrm{dL}$ & $\begin{array}{l}\text { Mayor o igual a } 150 \mathrm{mg} / \mathrm{dL} \text { ó estar recibiendo } \\
\text { tratamiento específico para esta dislipidemia }\end{array}$ \\
\hline Colesterol HDL & $\begin{array}{l}\text { Menor de } 50 \mathrm{mg} / \mathrm{dL} \text { en mujeres y } 40 \\
\mathrm{mg} / \mathrm{dL} \text { en hombres }\end{array}$ & $\begin{array}{l}\text { Menor de } 50 \mathrm{mg} / \mathrm{dL} \text { en mujeres y } 40 \mathrm{mg} / \mathrm{dL} \\
\text { en hombres ó estar recibiendo tratamiento } \\
\text { específico para esta dislipidemia }\end{array}$ \\
\hline Presión arterial & $\begin{array}{l}\text { Presión arterial mayor o igual a } \\
130 / 85 \mathrm{mmHg} \text { o tener diagnóstico } \\
\text { previo de HTA y estar recibiendo } \\
\text { medicamentos }\end{array}$ & $\begin{array}{l}\text { Sistólica } \geq 130 \mathrm{mmHg} \text { o diastólica } \geq 85 \\
\mathrm{mmHg} \text { ó estar en tratamiento de hipertensión } \\
\text { diagnosticada previamente }\end{array}$ \\
\hline Glucemia & $\begin{array}{l}\text { Glucemia en ayunas mayor o igual } \\
\text { a } 110 \mathrm{mg} / \mathrm{dL} \text { ó tener diagnóstico } \\
\text { previo de diabetes y estar } \\
\text { recibiendo tratamiento }\end{array}$ & $\begin{array}{l}\text { Glicemia en ayunas } \geq 100 \mathrm{mg} / \mathrm{dL} \text { ó diagnóstico } \\
\text { previo de diabetes tipo } 2\end{array}$ \\
\hline
\end{tabular}

NCEP: National Cholesterol Education Program (26). IDF: International Diabetes Federation (27).

La asociación entre el SM y la inflamación está bien documentada (34). La evidencia acumulada demuestra una estrecha relación entre el síndrome metabólico, un estado de inflamación crónica y estrés oxidativo (35). El aumento del estrés oxidativo ha surgido como un componente que juega un papel central en la explicación del SM y sus patologías adyacentes, además de ser un factor elemental en la progresión de esta enfermedad. Las especies reactivas de oxígeno (ROS) son derivadas del metabolismo del oxígeno altamente reactivo. Estas moléculas juegan papeles importantes en procesos fisiológicos normales tales como la expresión génica y la transducción de señales. En un estado saludable, las ROS se mantienen a un nivel óptimo debido a un equilibrio entre su producción y eliminación por antioxidantes enzimáticos (superóxidodismutasa, glutatión, catalasa, peroxidasa) y no enzimáticos (vitaminas $C$ y E). En un estado patológico tal como el SM, un aumento de la capacidad oxidante junto con una capacidad antioxidante disminuida crea un desequilibrio que se traduce en estrés oxidativo (36).

El aumento de los niveles de ROS durante el estrés oxidativo tiene efectos tóxicos sobre células y tejidos a través del aumento de la oxidación de carbohidratos, lípidos y proteínas. Se ha demostrado que desempeñan un papel importante en el desarrollo y progresión de la enfermedad cardiovascular; además ha sido identificado como un importante mecanismo de complicaciones micro y macrovasculares en el SM (37). De hecho, la vía de la inflamación en el estrés oxidativo tiene importantes funciones en todos los componentes individuales del SM, incluyendo alteraciones vasculares (38).

EI ATP III ha puesto un mayor énfasis en los cambios de estilo de vida terapéuticos como una estrategia esencial para el manejo clínico de las personas en riesgo de enfermedad cardiovascular. Los cambios en el comportamiento alimenticio y el ejercicio pueden dar lugar a un índice de masa corporal (IMC) saludable, mejores valores de HDL y triglicéridos, reducir la presión arterial y bajar la glicemia. Los cambios en el estilo de vida deben ser una parte integral de la terapia de reducción de riesgo para el SM y la diabetes mellitus tipo 2 . Sin embargo, estas recomendaciones se centran principalmente en pacientes con factores de riesgo específicos para el desarrollo de síndrome metabólico, como obesidad, diabetes, presión arterial alta, enfermedad coronaria, entre otros $(39,40)$. 


\section{MENOPAUSIA Y ENFERMEDAD CARDIOVAS- CULAR}

La menopausia es una etapa muy importante ya que en ella convergen una serie de factores de riesgo que pueden agudizarse. Cerca del $60 \%$ de las mujeres menopáusicas tienen una enfermedad crónica y son las enfermedades cardiovasculares (ECV) las principales causas de morbilidad y mortalidad a partir de esta época (41), lo cual podría estar relacionado con los cambios metabólicos que ocurren durante la transición de un estado pre a uno post menopáusico (41).

Generalmente la ECV comienza 10 años más tarde en las mujeres que en los hombres y es raro observar un infarto agudo del miocardio en el sexo femenino antes de los 50 años (42), lo que hace pensar que las mujeres premenopáusicas están protegidas para ECV, en comparación con hombres de la misma edad. Sin embargo, a los 70 años la incidencia de estas enfermedades es igual en ambos sexos, lo que sugiere que la deficiencia de estrógenos causa una rápida aceleración en el riesgo de presentar enfermedades cardiacas y vasculares (42).

A pesar de esto, aún existe controversia sobre la razón por la cual la menopausia incrementa el riesgo cardiovascular independiente de la otras variables sociodemográficas como la edad. Algunos estudios han demostrado incremento del riesgo de ECV después de la menopausia y otros no (43). El estudio Framingham (44), encontró 4 veces mayor prevalencia de ECV en los 10 años siguientes a la menopausia. La menopausia prematura inducida quirúrgicamente también se ha relacionado con incremento del riesgo cardiovascular (43).

Al hablar de riesgo cardiovascular en este contexto, resulta difícil separar los efectos fisiológicos de la edad de los procesos mediados por el déficit estrogénico de la menopausia. Diversos estudios que han evaluado la relación entre menopausia y ateroesclerosis han encontrado diversos resultados. Sutton-Tyrrell y cols (45), mostró que el $45 \%$ de las mujeres posmenopáusicas tienen engrosamiento de la íntima en la carótida con significado clínico, comparado con el $16 \%$ de las mujeres premenopáusicas; este engrosamiento de la íntima ha mostrado ser un buen predictor de riesgo de ECV (46). Otras medidas como la determinación de ateroesclerosis en la aorta, la extensión de su calcificación y los depósitos de calcio en las arterias coronarias, han mostrado mayores índices en mujeres posmenopáusicas (47).

\section{RELACIÓN ENTRE SÍNDROME METABÓLICO Y MENOPAUSIA}

En la última década, se ha presentado un aparente aumento de la prevalencia de obesidad en la mayoría de las mujeres, incrementando a la vez la frecuencia del SM en esta misma población. Debido a que se ha asociado este síndrome al aumento de la edad, muchos autores han reportado este fenómeno especialmente en la transición de la etapa pre a la postmenopausia (48). SM ha sido considerado un valor predictivo de la enfermedad cardiovascular en las mujeres, a diferencia de la población masculina donde no se haya relación. De igual forma se ha encontrado que la menopausia se encuentra ligada a algunos componentes de este síndrome y deja la inquietud del comportamiento de estas dos condiciones y su interacción (49).

La transición que experimenta la mujer durante la pre y postmenopausia, se asocia con la presentación o el desarrollo de características propias del SM, entre las que se encuentran (50): aumento de la grasa central abdominal, alteración del perfil lipídico (aumento de lipoproteínas de baja densidad, los triglicéridos, disminución lipoproteínas de alta densidad y poca alteración de partículas de densidad intermedia, lo que lleva a la mayor incidencia de aterogénesis), y resistencia a la insulina. Por esta razón, la prevalencia de SM se incrementa con la menopausia hasta en un $60 \%$, al mezclarse variables como la edad, IMC, inactividad física entre otras $(51,52)$. Este fenómeno puede explicar parcialmente el incremento en la incidencia de ECV observado después de la menopausia. Lo anterior se podría explicar como resultado directo de la falla ovárica o indirectamente por consecuencias metabólicas de la distribución central de la grasa asociada a la deficiencia estrogénica (50-52).

La menopausia se asocia con ganancia de peso e incremento de la adiposidad abdominal, independiente de la edad y de la grasa corporal; este acumulo de grasa visceral es el mayor determinante del SM. Diferencias en la actividad de la lipoproteína lipasa en la grasa acumulada pueden determinar aun mayor acumulo de grasa. La adiponectina por su parte, un péptido derivado de los adipocitos, juega un papel muy importante en este síndrome, ya que su concentración es inversamente proporcional con la obesidad y con la resistencia periférica a la insulina (53-55).

La menopausia también se relaciona con disminución de la masa muscular secundaria a la disminución de la actividad física, la cual lleva a menor 
consumo de oxígeno y por ende a un incremento de la adiposidad central $(56,57)$. La grasa de distribución central se asocia con anormalidades en los lípidos, resistencia a la insulina, elevación en los niveles de ácidos grasos libres y disminución de la adiponectina, fenómenos que contribuyen al incremento en la secreción de apolipoproteina B y por ende, a la presentación de hipertrigliceridemia (16\%), asociado a un incremento de la actividad de la lipasa hepática, disminución de las partículas antiaterogénicas como las lipoproteínas de alta densidad (HDL) (25\%) y un aumento de las lipoproteínas de baja densidad (LDL), las cuales pasan del 10$13 \%$ al $30-49 \%$ durante la transición menopaúsica. Esto en la clínica se ve reflejado en una mayor incidencia de infarto agudo del miocardio, así como su relación con mayor severidad de estas enfermedades cardiovasculares (58).

La resistencia a la insulina se asocia con hiperinsulinemia, la cual lleva a una inadecuada supresión de los ácidos grasos libres en los tejidos y altera la captación de la glucosa a nivel periférico. Diversos estudios han evidenciado que el estado postmenopáusico se asocia con elevación de los niveles de insulina y glucosa más que en el estado premenopáusico, lo que empeoraría aún más la resistencia a la insulina $(59,60)$. Las adipocitoquinas circulantes, liberadas por los depósitos de grasa, están implicadas en los mecanismos generadores de la resistencia a la insulina y la ECV. En mujeres posmenopáusicas con SM se ha encontrado aumento de las leptinas, asociado a resistencia y reducción en las adiponectinas, las cuales tienen un efecto protector (61).

Por otro lado, la menopausia tiene efectos en los niveles de lípidos plamáticos, evidenciándose un aumento significativo de las LDL, colesterol total y triglicéridos, asociados al descenso de las HDL $(62,63)$. Yoldemir y cols $(62)$, reportaron que el aumento en la LDL fue menor en mujeres premenopáusicas que en las posmenopáusicas, al igual que el aumento en los triglicéridos, la disminución del HDL y la obesidad central, siendo el HDL y los triglicéridos los de mejor correlación en la población turca.

Ebrahimpour y cols (48), encontraron que la prevalencia de los factores de riesgo cardiovascular en población Iraní fue mayor en las mujeres postmenopaúsicas, con diferencia significativa al compararlas con las premenopáusicas. El porcentaje de pacientes con SM fue del $60 \%$, diferenciándose de la población masculina con un porcentaje tan solo del 19\%. Varios estudios han evidenciado, de forma concordante con estos hallazgos, una alta prevalencia de SM en mujeres posmenopáusicas según criterios NCEP/ATPIII; se enuncian entonces prevalencias del 26,1\% en Colombia (64), 31\% en México (65) y Canadá (66), 36,1\% en Alemania (67), 42,2\% en Brasil (68), 50,5\% en Ecuador (69) y $54,6 \%$ en Corea del Sur (70).

Los hallazgos de algunas investigaciones sugieren que, en las mujeres posmenopáusicas, la presencia de SM podría estar relacionada con el riesgo de cáncer de mama (71). Rohany cols (72), utilizaron absorciometría de rayos $X$ de energía dual (DXA) para determinar medidas derivadas de grasa corporal y examinar la asociación entre estas y el riesgo de cáncer de mama. El estudio incluyó a 10960 mujeres posmenopáusicas de 50 a 79 años sin antecedentes de cáncer de mama. Durante un seguimiento de 12,9 años en promedio, se diagnosticaron 503 casos de cáncer de mama. Índices antropométricos DXA como el índice de masa corporal, la circunferencia de la cintura y la relación cintura/cadera mostraron asociaciones fuertemente positivas con el riesgo de padecer este tipo de cáncer (72).

\section{CONCLUSIÓN}

La verdadera importancia de los factores que influyen en el riesgo cardiovascular en las mujeres posmenopáusicas está lejos de determinarse con exactitud y total certeza. Las alteraciones en el metabolismo de los lípidos por deficiencia estrogénica es un componente del riesgo cardiovascular, sin embargo efectos directos de los cambios hormonales sobre la distribución de la grasa corporal, la acción de la insulina, la pared arterial y la fibrinolisis pueden impactar en la salud de las mujeres. Estos factores contribuyen a incrementar la prevalencia de SM en la postmenopausia, el cual, asociado al empeoramiento del perfil metabólico, puede contribuir al riesgo de presentar enfermedades cardiovasculares con mayor frecuencia y severidad. Los cambios anteriormente descritos durante la menopausia a nivel metabólico nos orientan a reconocer la importancia de identificar aquellas mujeres que desarrollan rasgos de SM, las cuales deberían ser tratadas en forma temprana e integral con el fin de reducir el riesgo de ECV. Dichas intervenciones varían desde modificaciones del estilo de vida hasta la implementación de medidas farmacológicas, las cuales deben ir siempre dirigidas al mejoramiento de la salud y de la calidad de vida de las mujeres en menopausia. 


\section{REFERENCIAS}

1. Okeke TC, Ezenyeaku CC, Ikeako LC, Agu PU. An overview of menopause associated vasomotor symptoms and options available in its management. Niger J Med 2013;22(1):7-14.

2. Jones EK, Jurgenson JR, Katzenellenbogen JM, Thompson SC. Menopause and the influence of culture: another gap for Indigenous Australian women? BMC Womens Health 2012;12:43.

3. Nelson HD. Menopause. Lancet 2008;371(9614):76070.

4. Utian WH, Woods NF. Impact of hormone therapy on quality of life after menopause. Menopause 2013;20(10):1098-105.

5. Gudmundsdottir SL, Flanders WD, Augestad LB. Physical activity and cardiovascular risk factors at menopause: The Nord-Trøndelag health study. Climacteric 2013;16(4):438-46.

6. Davis SR, Castelo-Branco C, Chedraui P, Lumsden MA, Nappi RE, Shah D, Villaseca P; Writing Group of the International Menopause Society for World Menopause Day 2012. Understanding weight gain at menopause. Climacteric 2012;15(5):419-29.

7. Gold EB, Crawford SL, Avis NE, Crandall CJ, Matthews KA, Waetjen LE, et al. Factors related to age at natural menopause: longitudinal analyses from SWAN. Am J Epidemiol 2013;178(1):70-83.

8. Pfister AK, Welch CA, Emmett MK, Sheets NW. Risk factors predicting fractures in early postmenopausal women. W V Med J2013;109(3):8-12, 14-5.

9. Madero JI, López C, León MF, Ruiz J, Gutiérrez C, Guerra O, Ávila M, et al. Importancia de la respuesta a la inducción de ovulación y la edad en el resultado de las técnicas de reproducción asistida. Rev Colomb Obstet Ginecol 2004;55(4):293-9.

10. Argote LA, Mejía ME, Vásquez ML, Villaquirán ME. Climaterio y menopausia en mujeres afrodescendientes: una aproximación al cuidado desde su cultura. Aquichan 2008;8(1):33-48.

11. Morris $\mathrm{E}$, Currie $\mathrm{H}$. Estrogen deficiency: Education for all! Menopause Int 2013;19(2):55.

12. Mishra N, Mishra VN, Devanshi. Exercise beyond menopause: Dos and Don'ts. J Midlife Health 2011;2(2):51-6.

13. Nelson HD, Walker M, Zakher B, Mitchell J. Menopausal hormone therapy for the primary prevention of chronic conditions: a systematic review to update the U.S. Preventive Services Task Force recommendations. Ann Intern Med 2012;157(2):10413.

14. Bassol-Mayagoitia S. La edad de la menopausia en México. RevEndocrinolNutr 2006;14(3):133-6.

15. Canto de Cetina T. Los síntomas en la menopausia. Rev Endocrinol Nutr 2006;14(3):141-8.

16. Mashtare ML, Lee LS, Nies L, Turco RF Transformation of 17a-estradiol, 17 $\beta$-estradiol, and estrone in sediments under nitrate- and sulfate-reducing conditions. Environ Sci Technol 2013;47(13):7178-85.

17. Brenseke B, Prater MR, Bahamonde J, Gutierrez
JC. Current thoughts on maternal nutrition and fetal programming of the metabolic syndrome. J Pregnancy 2013;2013:368461.

18. Parkinson JR, Hyde MJ, Gale C, Santhakumaran S, Modi N. Preterm birth and the metabolic syndrome in adult life: a systematic review and meta-analysis. Pediatrics 2013;131(4):e1240-63.

19. Hutcheson R, Rocic P. The metabolic syndrome, oxidative stress, environment, and cardiovascular disease: the great exploration. Exp Diabetes Res 2012;2012:271028.

20. Suzuki T, Hirata K, Elkind MS, Jin Z, Rundek T, Miyake $\mathrm{Y}$, et al. Metabolic syndrome, endothelial dysfunction, and risk of cardiovascular events: the Northern Manhattan Study (NOMAS). Am Heart J 2008;156(2):405-10.

21. Ärnlöv J, Ingelsson E, Sundström J, Lind L. Impact of body mass index and the metabolic syndrome on the risk of cardiovascular disease and death in middleaged men. Circulation 2010;121(2):230-6.

22. Noda H, Iso H, Saito I, Konishi M, Inoue M, Tsugane S; JPHC Study Group. The impact of the metabolic syndrome and its components on the incidence of ischemic heart disease and stroke: the Japan public health center-based study. Hypertens Res 2009;32(4):289-98.

23. Yamaguchi $T$, Kitamori K, Ichihara G, Suzuki $Y$, Ochiai M, Yamada $Y$, et al. Serial changes in adipocytokines and cardiac function in a rat model of the metabolic syndrome. Clin Exp Pharmacol Physiol 2013;40(7):443-8.

24. Renna NF, Diez ER, Lembo C, Miatello RM. Role of Cox-2 in vascular inflammation: an experimental model of metabolic syndrome. Mediators Inflamm 2013;2013:513251.

25. Morange PE, Alessi MC. Thrombosis in central obesity and metabolic syndrome: Mechanisms and epidemiology. Thromb Haemost 2013;110(4):669-80.

26. Executive summary of the Third Report of the National Cholesterol Education Program (NCEP) Expert on detection, evaluation, and treatment of high blood colesterol in adults (Adult Treatment Panel III). JAMA 2001;285(19):2486-97.

27. International Diabetes Federation. The IDF consensus worldwide definition of the metabolic syndrome, 2005. Disponible en: http://www.idf.org/webdata/docs/ Metac_syndrome_def.pdf

28. Santaniemi M, Ukkola O, Malo E, Bloigu R, Kesäniemi YA. Metabolic syndrome in the prediction of cardiovascular events: The potential additive role of hsCRP and adiponectin. Eur J PrevCardiol 2013. [Epub ahead of print].

29. Reaven GM. Insulin resistance: the link between obesity and cardiovascular disease. Med Clin North Am 2011;95(5):875-92.

30. Aguilar-Salinas CA, García EG, Robles L, Riaño D, Ruiz-Gomez DG, García-Ulloa AC, Melgarejo MA, Zamora M, Guillen-Pineda LE, Mehta R, CanizalesQuinteros S, Tusie Luna MT, Gómez-Pérez FJ. High adiponectin concentrations are associated with the metabolically healthy obese phenotype. J Clin Endocrinol Metab 2008;93(10):4075-9. 
31. Stefan N, Kantartzis K, Machann J, Schick F, Thamer C, Rittig K, Balletshofer B, Machicao F, Fritsche A, Häring $\mathrm{HU}$. Identification and characterization of metabolically benign obesity in humans. Arch Intern Med 2008;168(15):1609-16.

32. Uretsky S, Messerli FH, Bangalore S, Champion A, Cooper-Dehoff RM, Zhou Q,Pepine CJ. Obesity paradox in patients with hypertension and coronary artery disease. Am J Med 2007;120(10):863-70.

33. Wildman RP, Muntner P, Reynolds K, McGinn AP, Rajpathak S, Wylie-Rosett J, Sowers MR. The obese without cardiometabolic risk factor clustering and the normal weight with cardiometabolic risk factor clustering: prevalence and correlates of 2 phenotypes among the US population (NHANES 1999-2004). Arch Intern Med 2008;168(15):1617-24.

34. Poelkens F, Lammers G, Pardoel EM, Tack CJ, Hopman MT. Upregulation of skeletal muscle inflammatory genes links inflammation with insulin resistance in women with the metabolic syndrome. Exp Physiol 2013;98(10):1485-94.

35. Yubero-Serrano EM, Delgado-Lista J, Peña-Orihuela $\mathrm{P}$, Perez-Martinez P, Fuentes F, Marin C, Tunez I, et al. Oxidative stress is associated with the number of components of metabolic syndrome: LIPGENE study. Exp Mol Med 2013;45:e28.

36. Lassègue B, Griendling KK. NADPH oxidases: functions and pathologies in the vasculature. Arterioscler Thromb Vasc Biol 2010;30(4):653-61.

37. Folli F, Corradi D, Fanti P, Davalli A, Paez A, Giaccari $A$, Perego $C$, Muscogiuri $G$. The role of oxidative stress in the pathogenesis of type 2 diabetes mellitus micro- and macrovascular complications: avenues for a mechanistic-based therapeutic approach. Curr Diabetes Rev 2011;7(5):313-24.

38. Simão TN, Lozovoy MA, Simão AN, Oliveira SR, Venturini D, Morimoto HK, et al. Reduced-energy cranberry juice increases folic acid and adiponectin and reduces homocysteine and oxidative stress in patients with the metabolic syndrome. $\mathrm{Br} \mathrm{J}$ Nutr 2013;110(10):1885-94.

39. Wilmot EG, Davies MJ, Edwardson CL, Gorely T, Khunti K, Nimmo M, et al. Rationale and study design for a randomised controlled trial to reduce sedentary time in adults at risk of type 2 diabetes mellitus: project stand (Sedentary Time ANd diabetes). BMC Public Health 2011;11:908.

40. Cheng AY, Leiter LA.Implications of recent clinical trials for the National Cholesterol Education Program Adult Treatment Panel III guidelines. Curr Opin Cardiol 2006;21(4):400-4.

41. Lwow F, Jedrzejuk D, Dunajska K, Milewicz A, Szmigiero L. Cardiovascular disease risk factors associated with low level of physical activity in postmenopausal Polish women. Gynecol Endocrinol 2013;29(7):683-6.

42. Go AS, Mozaffarian D, Roger VL, Benjamin EJ, Berry JD, Blaha MJ, et al; American Heart Association Statistics Committee and Stroke Statistics Subcommittee. Heart disease and stroke statistics--2014 update: a report from the American Heart Association. Circulation. 2014;129(3):e28-e292.
43. Lejsková M, Alušík $S$, Valenta Z, Adámková $S$, Pitha $\mathrm{J}$. Natural postmenopause is associated with an increase in combined cardiovascular risk factors. Physiol Res 2012;61(6):587-96.

44. Trikudanathan S, Pedley A, Massaro JM, Hoffmann $\mathrm{U}$, Seely EW, Murabito JM, et al. Association of female reproductive factors with body composition: the Framingham Heart Study. J Clin Endocrinol Metab 2013;98(1):236-44.

45. Sutton-Tyrrell K, Lassila, HC, Meilahn E, Bunker C, Matthews KA, Kuller LH. Carotid atherosclerosis in premenopausal and postmenopausal women and its association with risk factors measured after menopause. Stroke 1998;29:1116-21.

46. Lock M. Symptom reporting at menopause: a review of cross-cultural findings. $\mathrm{J} \mathrm{Br}$ Menopause Soc 2002;8(4):132-6.

47. Sumino $H$, Murakami M. Investigation of atherosclerosis in postmenopausal women: alteration of atherosclerosis-associated factors and vascular atherosclerosis by oral and transdermal estrogen replacement. Rinsho Byori 2013;61(3):256-62.

48. Ebrahimpour P, Fakhrzadeh H, Heshmat R, Ghodsi M, Bandarian F, Larijani B. Metabolic syndrome and menopause: A population-based study. Diabetes \& Metabolic Syndrome: Clinical Research \& Reviews. 2010;4:5-9.

49. Eshtiaghia R, Esteghamatib A, Nakhjavani M. Menopause is an independent predictor of metabolic syndrome in Iranian women. Maturitas 2010;65(3):262-6.

50. Jouyandeh Z, Nayebzadeh F, Qorbani M, Asadi M. Metabolic syndrome and menopause. J Diabetes Metab Disord 2013;12(1):1.

51. Ross LA, Polotsky AJ. Metabolic correlates of menopause: an update. Curr Opin Obstet Gynecol 2012;24(6):402-7.

52. van der Leeuw J, Wassink AM, van der Graaf $Y$, Westerveld HE, Visseren FL; on behalf of the Second Manifestations of ARTerial Disease (SMART) Study Group. Age-related differences in abdominal fat distribution in premenopausal and postmenopausal women with cardiovascular disease. Menopause 2013;20(4):409-17.

53. Thurston RC, Chang $Y$, Mancuso $P$, Matthews KA. Adipokines, adiposity, and vasomotor symptoms during the menopause transition: findings from the Study of Women's Health Across the Nation. Fertil Steril 2013;100(3):793-800.

54. Matsui S, Yasui T, Tani A, Kato T, Kunimi K, Uemura H, et al. Difference in the Ratio of High-Molecular Weight (HMW) to Total Adiponectin and HMW Adiponectin in Late Postmenopausal Women. J Endocrinol Invest 2013;36(11):982-5.

55. Mascarenhas-Melo F, Marado D, Palavra F, Sereno $\mathrm{J}$, Coelho Á, Pinto R, et al. Diabetes abrogates sex differences and aggravates cardiometabolic risk in postmenopausal women. Cardiovasc Diabetol 2013;12:61.

56. Barbat-Artigas S, Aubertin-Leheudre M. Menopausal transition and fat distribution. Menopause 2013;20(4):370-1. 
57. Ross LA, Polotsky AJ. Metabolic correlates of menopause: an update. CurrOpinObstetGynecol 2012;24(6):402-7.

58. Berg GA, Siseles N, González Al, Ortiz OC, Tempone A, Wikinski RW. Highervalues of hepatic lipase activity in postmenopause: relationship with atherogenic intermediate density and low density lipoproteins. Menopause 2001;8(1):51-7.

59. Monterrosa-Castro A, Blümel JE, Portela-Buelvas $\mathrm{K}$, Mezones-Holguín E, Barón G, Bencosme A, et al; for the Collaborative Group for Research of the Climacteric in Latin America (REDLINC). Type II diabetes mellitus and menopause: a multinational study. Climacteric 2013;16(6):663-72.

60. Peppa M, Koliaki C, Papaefstathiou A, Garoflos E, Katsilambros N, Raptis SA, et al. Body composition determinants of metabolic phenotypes of obesity in nonobese and obese postmenopausal women. Obesity (Silver Spring). 2012;21(9):1807-14.

61. Lobo RA. Metabolic syndrome after menopause and the role of hormones. Maturitas 2008;60(1):10-8.

62. Yoldemir T, Erenus M. The prevalence of metabolic syndrome in pre-and post-menopausal women attending a tertiary clinic in Turkey. Eur $\mathrm{J}$ Obstet Gynecol Reprod Biol 2012;164(2):172-5.

63. Ainy E, Mirmiran P, ZahediAsI S, Azizi F. Prevalence of metabolic syndrome during menopausal transition Tehranian women: Tehran Lipid and Glucose Study (TLGS). Maturitas 2007;58(2):150-5.

64. Sánchez $F$, Jaramillo $N$, Vanegas $A$, Echeverri JG, Alviar CL, Echavarría E, et al. Prevalencia y comportamiento de los factores de riesgo del síndrome metabólico según los diferentes intervalos de edad, en una población femenina del área de influencia de la clínica Las Américas, en Medellín Colombia. Rev Colomb Cardiol 2008;15:102-10.

65. Tabares MK, Aguilera JR, Velázquez B, Garza P, Angulo LC, García R. Síndrome metabólico en menopausia: implicaciones de la terapia hormonal. Perinatol Reprod Hum 2012;26(1):25-9.

66. Piché ME, Weisnagel SJ, Corneau L, Nadeau A, Bergeron J, Lemieux $\mathrm{S}$. The WHO and NCEP/ ATPIII definitions of the metabolic syndrome in postmenopausal women: are they so different? Metab Synd rRelat Disord 2006;4(1):17-27.

67. Deibert P, König D, Vitolins MZ, Landmann U, Frey I, Zahradnik HP, et al. Effect of a weight loss intervention on anthropometric measures and metabolic risk factors in pre- versus postmenopausal women. Nutr $\mathrm{J}$ 2007;6: 31

68. Schmitt AC, Cardoso MR, Lopes H, Pereira WM, Pereira EC, de Rezende DA, et al. Prevalence of metabolic syndrome and associated factors in women aged 35 to 65 years who were enrolled in a family health program in Brazil. Menopause 2013;20(4):4706.

69. Chedraui P, San Miguel G, Villacreses D, Dominguez A, Jaramillo W, Escobar GS, et al; Research Group for the Omega Women's Health Project. Assessment of insomnia and related risk factors in postmenopausal women screened for the metabolic syndrome. Maturitas 2013;74(2):154-9.

70. Kim HM, Park J, Ryu SY, Kim J. The effect of menopause on the metabolic syndrome among Korean women: the Korean National Health and Nutrition Examination Survey, 2001. Diabetes Care 2007;30(3):701-6.

71. Esposito K, Chiodini P, Capuano A, Bellastella G, Maiorino MI, Rafaniello C, et al. Metabolic syndrome and postmenopausal breast cancer: systematic review and meta-analysis. Menopausia 2013;20(12):1301-9.

72. Rohan TE, Heo M, Choi L, Datta M, Freudenheim JL, Kamensky V, et al. Body fat and breast cancer risk in postmenopausal women: a longitudinal study. J CancerEpidemiol 2013;2013:754815. 